\title{
Trends in Managing Parkinson's Disease - A Review
}

\author{
SM RASHID HILALY ${ }^{1}$, MD. TAUHIDUL ISLAM CHOWDHURY², MOHAMMAD SHAH JAHIRUL HOQUE CHOWDHURY ${ }^{3}$
}

\begin{abstract}
:
Natural fluidity of movement \& feelings seem to be lost in PD. Automatic activities of facial expression, swallowing, speaking, walking become less automatic. Clinical experience is essential to diagnose, decide symptom worsening, determine end of dose wearing off \& the on-off states, acknowledge non-motor problems, titrate dopaminergic treatment \& add adjunctive treatment. Current breakthroughs in the treatment concepts like discovery of non-dopaminergic brain targets, neuroplasticity, L-DOPA formulations including Levodopa/carbidopa intestinal gel[ $[C I G]$, pump therapies, deep brain stimulation have been discussed. LCIG is available in some 30 countries but cost makes it prohibitive for widespread use. Goalbased motor skill training improves cognitive \& automatic components of motor control in mild to moderate PD. Levodopa + carbidopa, remains the gold standard \& eventually leads to L-DOPA induced dyskinesia in the majority within 10 years of treatment. MAO-B inhibitors have an excellent side effect profile but mild motor benefit. Dopamine agonists (nonergot-ropinirole, pramipexole) provides moderate symptomatic benefit and delay the development of dyskinesia but at the expense of psychiatric and nonmotor side effects.
\end{abstract}

Keywords: Parkinson's disease, nonmotor symptoms, motor symptoms, neuroplasticity, direct pathway, Orthostatic hypotension, Constipation, Sialorrhea, DDS, ICD, Punding.

\section{Introduction:}

For a diagnosis which is largely clinical \& a management which has many schools of thought regarding the initiation of treatment $\&$ adjuvant therapy the goal of management is focused on optimizing patients quality of life while minimising adverse effects of drugs. Fundamental to the diagnosis are: Bradykinesia-slowness of initiation of voluntary movement with progressive fatiguing, decrement of repetitive alternating movements during ûnger or foot tapping. Resting tremor \& rigidity on passive movement. Initially unilateral eventually progressive, bilateral \& subsequently postural instability (not from primary visual, vestibular, cerebellar or proprioceptive dysfunction), falls, orthostatic hypotension and dementia can develop. Recently, hyposmia and visual hallucinations have been added. Exclusion criteria for Parkinson's disease[PD] need to be checked [Queen Square Brain Bank UK PDS Brain Bank Criteria for the diagnosis of $\mathrm{PD}] \cdot{ }^{1,2}$

1. Former Professor of Internal Medicine, Nightingale Medical College \& Jahurul Islam Medical College, Dhaka University.

2. Assistant Professor(Neurology), National Institute of Neurosciences \& Hospital, Sher-e-Bangla Nagar, Dhaka

3. Assistant Professor cum Resident Physician, National Institute of Neurosciences \& Hospital, Sher-e- Bangla Nagar, Dhaka

Correspondence : Prof. SM Rashid Hilaly, Former Professor of Internal Medicine, Nightingale Medical College \& Jahurul Islam Medical College, Dhaka University. lectincdl@gmail.com

\section{Four common presentations ${ }^{7}$ :}

- tremor,

- a weak and clumsy limb,

- a stiff and aching limb, and

- a gait disorder

There is no ideal way to define PD and distinguish it from other parkinsonian syndromes. Nevertheless, PD needs to be differentiated from mimics ${ }^{3,16}$ :

\begin{tabular}{ll}
\hline Degenerative disorders & Non-degenerative disorders \\
\hline Multiple system atrophy(MSA) & Essential tremor \\
Progressive supranuclear palsy(PSP) & Dystonic tremor \\
Corticobasal degeneration(CBD) & Cerebrovascular disease \\
Dementia with Lewy bodies & Drug-induced parkinsonism \\
Alzheimer's disease & \\
MPTP-induced parkinsonism & \\
\hline
\end{tabular}

Diagnostic error rate in a community sample was $47 \% .{ }^{4}$ So suspected patients should be referred, early \& untreated, to a specialist with sufficient expertise in movement disorders ${ }^{16}$. It is difficult even for experts to differentiate essential tremor from PD when asymmetric postural and action tremor of the upper limbs appears at rest. In this situation, ${ }^{123}$ I-FP-CIT SPECT should be considered. ${ }^{5}$ Intravenous isotope tagged to a cocaine derivative binds to the presynaptic dopamine reuptake site in the striatum, which is visualized using a gamma camera. Uptake is reduced in those with PD, PD dementias, and Parkinsonian syndromes. ${ }^{2}$ Routine use of 
functional imaging is not recommended for the differential diagnosis of Parkinson's disease and Parkinson's plus disorders such as progressive supranuclear palsy and multiple system atrophy. ${ }^{16}$ Objective olfactory testing is not recommended in the diagnosis of Parkinson's disease. ${ }^{16}$

Functional Neuroanatomy \& Basal Ganglia Circuitry: Motor function is not just locomotion but also the subtle automatic motor activities \& adjustments that give us a smooth flow in speech, swallowing, communicating body language, vision, writing, playing musical instruments.... Basal ganglia and cerebellum modulate the activity of motor cortex and the descending motor pathways. The basal ganglia are subcortical nuclei:

1. Striatum [input nucleus]

a. Caudate and putamen[neostriatum]

b. Accumbens

2. Globus Palidus[GP]

a. Internal segment[GPi] [output nucleus]

b. External segment[GPe]

3. Substantia nigra[SN]

a. pars compacta[SNc]

b. pars reticulate[SNr] [output nucleus]

4. Subthalamic nucleus[STN]

The action of basal ganglia is inhibitory. The output nuclei of the basal ganglia discharge tonically at high frequency to inhibit their target nuclei in the thalamus. Major inputs to the neostriatum are:

1. Glutamatergic input from sensory, motor, and association cortex [Fig1].

2. Glutamatergic input from the thalamus [Fig1].

3. Dopaminergic inputs from the SNc [Fig1].

4. Serotoninergic inputs from the dorsal raphe nucleus.

\section{Modulation of signals in the basal ganglia}

Direct \& indirect pathways process signals through the basal ganglia [Fig.-1] having opposite net effects on thalamus.

1. Excitation of the direct pathway ultimately disinhibits the thalamic neurons i.e. exciting thalamic neurons (which in turn make excitatory connections onto cortical neurons). Activation of the direct pathway would increase the ease of movement and of initiating movement.

2. Excitation of the indirect pathway has the net effect of inhibiting thalamic neurons (making them unable to excite motor cortex neurons).
Direct pathway selectively facilitates certain motor signals in the cerebral cortex that are needed for the present task, whereas the indirect pathway simultaneously inhibits the execution of competing motor activities. Imbalance between the direct and indirect pathways results in the motor dysfunctions.

\section{The nigrostriatal projection}

An important pathway in the processing of the direct and indirect pathways is the dopaminergic, projection from the $\mathrm{SNc}$ to the striatum Fig 1.

1. Direct pathway striatal neurons have D1 dopamine receptors, which depolarize the cell in response to dopamine. Thus exciting the direct pathway.

2. In contrast, indirect pathway striatal neurons have D2 dopamine receptors, which simultaneously inhibiting the indirect pathway.

The basal ganglia are also important in anatomical circuits that are involved in modulating non-motor aspects of behavior. Thus, the variation of cognitive and emotional functions in PD.

\section{Neurotransmitters}

- Glutamate are neurotransmitters having excitatory effects on their targets.

- GABA are neurotransmitters having inhibitory effects on their targets.

- $\quad$ Dopamine excitatory on D1 receptor \& inhibitory on D2 receptor.

- The striatum has one of the highest acetylcholine concentrations of any brain structure.

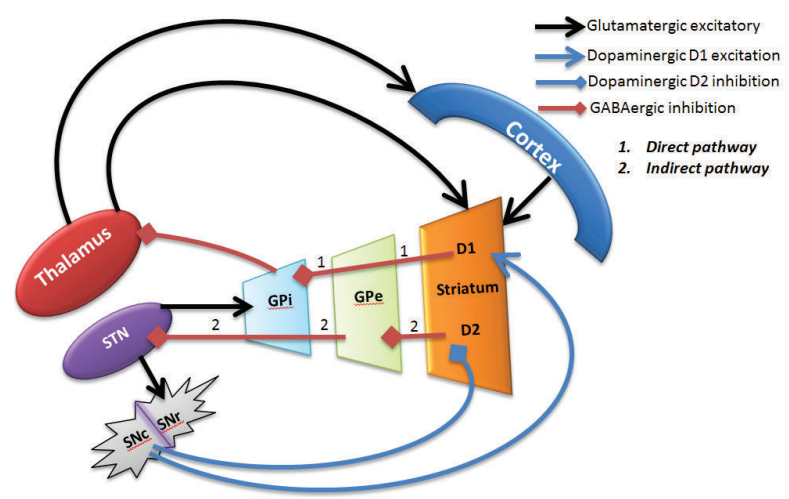

Fig.-1: Simplified Basal Ganglia Circuitry in normal condition - Hilaly et al.

\section{Direct pathway[Fig 1]:}

Glutamate from Cortex stimulates Striatum + Dopamine from SNc stimulates D1 in Striatum both inhibits GPi resulting in 
less inhibition of thalamus. So Thalamus stimulates the Cortex which stimulates the Muscles needed for the present task via lateral spinothalamic tract.

\section{Indirect pathway [Fig 1]:}

Cortex stimulates Striatum which inhibits GPe resulting in less inhibition of STN. So STN stimulates GPi which inhibits Thalamus\& Thalamus stimulates the Cortex less \& Muscles not stimulated brining a hypokinetic state. But Dopamine from SNc stimulates D2 simultaneously inhibits the indirect pathway \& decreases the hypokinetic state helping a smooth flow of movement.

\section{MANAGEMENT}

In spite of reaching an incontrovertible diagnosis the emerging therapeutic options \& cocktails used often makes the disease management complicated. For the last 200 years PD is a recognized neurodegenerative disorder withgradual loss of dopaminergic neurotransmission starting in substantia nigra which eventually spreads to the whole of the central nervous system.Disease modifying drugs still await to evolve. Symptomatic treatments available for PD include

- medications,

- $\quad$ surgical procedures,

- $\quad$ physiotherapy, occupational therapy and other support services.

all have a significant impact on improving an affected individual's quality of life. As disease progresses PD patients become more reliant on their medication to maintain mobility. A balance between the side effects versus medication benefit often becomes more difficult with time. Medication schedules become more complex and the timing of medications becomes pivotal.

Attempts at starting treatment very early in the disease, stems from the concept of delaying the progression. Clinical trials of proposedneuroprotective compounds have in general been unrewarding, although some compounds show promise. Further trials are currently underway. ${ }^{3}$ There have been promising results in animal models with selegiline, rasagiline, pramipexole and coenzyme Q10. To date, however, few neuroprotectiveresults were reproducible in humans. An option for younger patients with only mild symptoms may be, $1 \mathrm{mg}$ rasagiline [MAO-B inhibitory] daily over 18 months, possibly delays clinical progression in early stages of PD. ${ }^{6}$ Evidence based NICE [2006]\& Canadian guidelines, ${ }^{20,12}$ Scottish Intercollegiate Guidelines[2010] don't advocate neuroprotective treatment yet.

The current breakthroughs in the concepts of treatment of PD are

- the discovery of brain targets beyond the domains of dopamine \& dopamine agonists, like NMDA receptor, adenosine A2A receptor, Metabotropic Glutamate Receptors antagonists.

- role of neuroplasticity in determining dopaminergic therapy

- emphasis on physical \& rehabilitative therapy with the increasing understanding of experience-dependent neuroplasticity

- acknowledging\& managing non-motor problems which could be equally or at times more debilitating than the well described motor problems.

- deep brain stimulation [DBS]

- $\quad$ LCIG

Like all great discoveries evidence based benefits take time to establish.

\section{Management of motor symptoms}

Treatment is individualized to optimize quality of life, minimize side effects.

'Diagnosis does not warrant starting drugs' - is the ongoing clinical practice, and decision to start drugs is relied upon:

- $\quad$ symptom severity,

- whether the symptoms affect the dominant hand,

- embarrassment,

- ability to continue working and/or participate in activities such as hobbies,

- costand patient preference. With very mild symptomspatient may choose to delay therapy.

\section{Trends may be changing}

- Arguments exist whether treatment should be initiated early for dopaminergic neuronal"sparing". 3

- $\quad$ Recent trials suggest that people who start drug treatment soon after being diagnosed respond better than those who delay treatment and this may be related to brain plasticity.

- Furthermore dopamine-dependent corticostriatal plasticity is being investigated \& drugs that target long duration response may be beneficial at this stage over those that produce short duration response paving the way for disease modification.

Agents available for initial therapy include levodopa[LDOPA] in combination with carbidopa, dopamine agonists [DA], selective monoamine oxidase B (MAO-B) inhibitors, anticholinergic agents and amantadine. Most evidence based guidelines recommend L-DOPA, DA \& MAO-B as the first line agents. Anticholinergic drugs should not be used 
as first line treatment in patients with $\mathrm{PD}^{16}$ because of their limited efficacy and the possibility of neuropsychiatric side effects. Anticholinergic medications provide good tremor relief in approximately $50 \%$ of patients but do not meaningfully improve bradykinesia or rigidity. Because tremor may respond to one anticholinergic medication but not another, a second anticholinergic agent usually can be tried if the first is not successful. These medications should be introduced at a low dose and escalated slowly to minimize adverse effects, which include memory difficulty, confusion, and hallucinations. Adverse cognitive effects are relatively common, especially in elderly persons.Anticholinergic agents are added if tremor is vexatious \& are contraindicated for patients with dementia.For almost a century, anticholinergics remained the only possible treatment for parkinsonism. With the introduction of L-DOPA and increasing awareness of cognitive adverse reactions of anticholinergic drugs, interest in their use waned and the number of clinical trials declined. Dopaminergic-cholinergic antagonism in striatal function is the rationale behind their use.

Guidelines from the American Academy of Neurology and the evidence-based review of the Movement Disorder Society indicate that initiating therapy with L-DOPA or a DA is reasonable. Randomized trials have not found controlledrelease preparations to be superior to immediate-release preparations as initial therapy. ${ }^{7}$ Drug interactions may be a cause for failure to respond e.g. concomitant treatment with metoclopramide or risperidone. DAare slightly less effective than L-DOPA. Prospective double blinded studies show one potential advantage of DAover L-DOPA, a lower risk, by a factor of two or three, of dyskinesia and motor fluctuations in the first four to five years of treatment, particularly in DAmonotherapy. Within a few years L-DOPA needs to be added to control progressive symptoms. DAare avoided in patients with dementia because of the risk of hallucinations. DAhave more neuropsychiatric adverse effects than LDOPA, including sleepiness, sleep attacks, hallucinations, impulse control disorders, edema \& orthostatic hypotension. However, these adverse effects resolve upon lowering the dose or discontinuing the medication. Echocardiography in patients receiving long-term treatment with pergolide suggests that restrictive valvular disease may be two to four times more common ${ }^{7} \&$ serosal fibrosis (pleural, pericardial and retroperitoneal). ${ }^{16}$ So non-ergot DA(ropinirole, pramipexole, and rotigotine) are preferable to the ergot DA. ${ }^{16}$ MAO-B inhibitors (selegiline and rasagiline) have fewer adverse effects with simple dose titration, but effects tend to be mild.
COMT inhibitors, for instance, have no intrinsic effect but increase plasmatic levels of levodopa. It has been proven efficacious for adjunct therapy with levodopa and for the treatment of motor fluctuations.Entacapone[particularly combined with L-DOPA] is widely applied in clinical practice, improving activities of daily living and reducing the "off" time in fluctuating patients. ${ }^{6}$ Research and development of the adenosine $A_{2 A}$ receptor selective antagonist are ongoing.

\section{Management of special motor symptoms}

- Dyskinesias and fluctuations

- Freezing of gait

- Camptocormia

In prolong L-DOPA therapy, random fluctuations of motor symptoms appear in majority of the responding patients. After 9 years of L-DOPA treatment, $\sim 90 \%$ of PD patients experience dyskinesia ${ }^{12}$ consisting of choreiform twisting \&turning movements.Quality of life of the patient \& caregiver deteriorates. Causes are still being looked into \& the footprints of our understanding are:

- may due to fluctuations of plasma level of levodopa. Peak levels synchronizing with mobility with dyskinesia in contrast \& low plasma levels with bradykinesia in combination with nonmotor fluctuations (dysautonomic, cognitive + psychiatric\& pain). Interference with absorption of levodopa by food and by competition between large neutral amino acids and levodopa for transport from plasma to the brain may be partly responsible. $^{8}$

- there may be Imbalance of dopamine receptor subtypes. Progressive depletion of endogenous dopaminergic signaling plus exogenous supply of L-DOPA induces profound changes in the neurotransmitter network of basal ganglia. An imbalance in D1 and D2 receptors, mainly expressed in the direct and indirect striatal output pathways, has been identified in dyskinetic nonhuman primates ${ }^{11}$. However role of D2, D3 \& D5 have been suggested by other investigators.

- as the severity of PD increases, the substantial dopaminergic depletion leads to further adaptive changes in the basal ganglia pathways, including altered function of nondopaminergic basal ganglia neurotransmitters, such as glutamate, GABA, and serotonin. Excessive glutamatergic signalling within the basal ganglia is implicated in the progression of PD and in the emergence of L-DOPA induced dyskinesia [LID]. ${ }^{11}$

- Recent studies suggested that the cerebello-thalamocortical circuit also contributes to the development of LID. $^{22}$ 


\section{So the management is not black $\&$ white.}

- $\quad$ Non-pharmacologic therapies improve quality of life.All PD patients should receive regular physical and/or speech therapy and should be motivated to regular exercise. ${ }^{6}$

- The "on" freezing can be treated by reducing levodopa medication.

- The more common "off” freezing, is typically aimed at improving "on" time treatments. Catechol-o-methyl transferase inhibitors may be considered for the reduction in 'off' time in patients with advanced PD who have motor fluctuations.Intermittent subcutaneous apomorphine or LCIG may be considered for the reduction in 'off' time in patients with advanced PD. ${ }^{16}$

- $\quad$ MAOB inhibitors, DA, amantadine, COMT inhibitors (e.g., combined with levodopa) provide more stable plasmatic levels and are therefore a good option. ${ }^{6}$ NICE guidelines recommend that amantadine should be used as an anti-dyskinesia agent.NMDA glutamate receptor antagonist amantadine may significantly diminish LID. ${ }^{9}$ Further pharmacological modulation of glutamatergic transmission is a key focus for current research.

- Drug holiday carries some risk and does not improve the efficacy of levodopa therapy or prevent the LID. ${ }^{10}$

- Botulinum toxin injections in certain cases of freezing \&camptocormia

- The implantation of electrodes for Deep Brain Stimulation for non responders.

\section{Management of Non motor problems/syptoms [NMS]}

NMS may precede PD by years [ $>90 \%$ of PD present with hyposmia or anosmia], be present in early disease \& inescapably contribute to the severe disability of advanced disease. Focus being on the motor problems we unknowingly becomeunaware of NMS, causing a poor quality of life. Patients not realizing the connection with PD fail to report to the doctor about sialorrhoea, sleep disturbance, urinary dysfunction, constipation, pain, hallucination, depression, anxiety, memory impairment etc.. In fact non-motor symptoms may have a more significant impact on the quality of life than the motor symptoms.

Sleep disorders: may be intrinsic to PD or may be a dose dependent side effect of dopaminergic medication. Low-dose dopamine agonists have been associated with insomnia, whereas higher doses can lead to excessive daytime sleepiness. ${ }^{13}$
- Insomnia with consequent daytime somnolence is the most common. Education regarding healthy sleep hygiene. Clonazepam may be useful. ${ }^{6}$ Selegiline and amantadine have stimulating properties and can induce problems with sleep initiationso should be given earlier in the day.

- $\quad$ Sleep attacks were first described in patients treated with pramipexole or ropinirole.

- The use of prolonged release DA should be considered, as a recent study using ropinirole prolonged release demonstrated improved subjective quality of sleep, reduced daytime sleepiness, and disappearance of sleep attacks in some PD patients ${ }^{13}$. Modafinil and melatonin are not recommended for the management of excessivedaytime sleepiness associated with Parkinson's disease. ${ }^{16}$

- $\quad$ Restless legs syndrome (RLS). Managed by maintaining regular sleep pattern, exercise, massaging legs, heating pads or ice packs. Medications are DA, L-DOPA, benzodiazepine, gabapentin, opioids, and pregabalin.

Autonomic dysfunctions:orthostatic hypotension, gastrointestinal problems(Nausea, Constipation) \& bladder and erectile dysfunction are common.

- Orthostatic hypotension: a fall of at least $20 \mathrm{mmHg}$ systolic and/or 10 $\mathrm{mmHg}$ diastolic pressure within three minutes of standing. BP should be measured after 15 minutes of supine rest, and thereafter every minute for five minutes while standing, with measurement of the pulse. ${ }^{14}$ Early onset symptomatic orthostatic hypotension is an exclusion criteria for idiopathic PD.

o Sleeping in head-up position ( $>30^{\circ}$ incline), thigh and abdominal compression bands/ support stockings.

o Fragmentation of meals, increased water (2-2.5 L/ day) and salt intake (.8 g or $150 \mathrm{mmol} / \mathrm{L})$.

o avoid low sodium, carbohydrate-rich meals, alcohol, high temperature \&valsalva.

o Fludrocortisone, midodrine, a selective peripherally acting á-adrenergic agent, and droxidopa, an orally active synthetic precursor of norepinephrine, pyridostigmine are options. Reduction in dopaminergic medication, if these medications are not tolerated or if orthostatic hypotension is severe. $^{13}$

- Constipation:

o exercise, adequate water intake, and diet including symbiotic yogurts containing Bifidobacterium, 
fructoligosaccharide, and bulking agents (fibers, psyllium, and polycarbophil). A dietary herb extract, Dai-kenchu-to. ${ }^{13}$

o Osmotic laxatives including magnesium sulfate and polyethylene glycol. Other laxatives include lubiprostone and macrogol. Serotonergic agents such as cisapride, mosapride citrate, tegaserod have been shown to be beneficial as well.

o Constipation in PD may be associated with focal dystonia of the puborectalis muscle, and botulinum toxin A injections to the puborectalis muscle have demonstrated clinical benefit.

o Reduction of dopaminergic medications, amantadine, and anticholinergics should also be considered if possible.

- Dysphagia in late stages of PD very disabling and potentially harmful, as malnutrition, dehydration, aspiration, or even asphyxia may occur. Management includes optimising dopaminergic therapy, injection of botulinum toxin. Percutaneous endoscopic gastrostomy may be a last resort. ${ }^{6}$

- Sialorrhoeaaffects $\sim 75 \%$ of patients with PD. ${ }^{15} \mathrm{PD}$ patients do not produce more saliva, problem is swallowing dysfunction. Sialorrhea is often treated with anticholinergics, but central side effects limit their usefulness.

o Glycopyrrolate(glycopyrronium bromide) is an anticholinergic drug with a quaternary ammonium structure not able to cross the blood-brain barrier in considerable amounts, is safe. ${ }^{15}$

o Botulinum toxin is a more focused treatment.

Neuropsychiatric dysfunction: Substantia nigra \& dopamine are important components involved in pleasant feeling, rewards, addiction. So a deficiency affects an individual negatively.

- Psychosis in PD refers to the combination of chronic hallucinations and delusions in the setting of otherwise clear senses. May be benign to terribly frightening to the patient. It is multifactorial[drug \& disease]. Treatment for chronic hallucinations:

o reduction of dopaminergic medications and discontinuation of anticholinergics or other drugs.

o If needed, antipsychotic medications may be used. Clozapine has demonstrated efficacy in a doubleblind placebo-controlled trial; however, in clinical practice quetiapine is preferred, though not proven more effective than placebo in clinical trials. ${ }^{13}$ Clozapine is associated with agranulocytosis and regular monitoring of blood counts weeklyfor the first 18 weeks followed fortnightly for the first year and then monthly. ${ }^{16}$

- $\quad$ Dopamine dysregulation syndrome [DDS] characterized by addiction, self-medication, and escalation of antiparkinsonian medication. ${ }^{13}$ May be accompanied by ICD.

- Impulse control disorders [ICD] such as hypomania, hypersexuality and/or gambling, and dysphoria. The prevalence of any ICD in PD patients on dopamine agonists ranges from 13.7 to $17.1 \%$.

- $\quad$ Punding refers to stereotypic, complex, and repetitive behavior involving meaningless activities (i.e., examining, sorting, collecting, arranging, dismantling objects) sometimes to the point of ignoring basic needs such as eating and sleeping. Patient may be irritable when interrupted, and can lead to social avoidance and isolation.

- $\quad$ physicians must be aware of these neuropsychiatric side effects of dopaminergic therapy and screen for them

- family/caregiver history input can be particularly helpful as patient may deny or be embarrassed

- review of the patient's mediations is warranted, followed by a systematic reduction or cessation of dopaminergic treatment.

o For ICDs, this would meanDA. If parkinsonism worsens, it may be prudent to concomitantly increase levodopa.

o In DDS, the strategy is reversed and levodopa is weaned first, with aincrease in DA treatment.

- counseling with both the patients and their families is important.

- Antidepressants for obsessive thoughts

- Antiandrogens to help decrease hypersexuality may be considered.

- A recent study found amantadine to be effective in the treatment of pathological gambling in PD. Deep brain stimulation to the subthalamic nucleus may allow dopaminergic drug reduction and therefore improvement in these symptoms although DDS and ICDs may worsen or develop for the first time after DBS surgery. ${ }^{13}$

- Patients should be warned about the potential for dopamine agonists to cause impulsecontrol disorders 
and excessive daytime somnolence and be informed of the implicationsfor driving/operating machinery. ${ }^{16}$

Dementia Cognitive decline is one of the most disabling symptoms in PD during the later stages. A reason might be progressive neurodegeneration with cortical cholinergic deficiency. Anticholinergics and tricyclic antidepressants should be replaced where possible as they deteriorate cognitive performance. Available therapies are cholinesterase inhibitors - rivastigmine, which have demonstrated efficacy in the treatment of cognitive impairment in PD. ${ }^{6}$

\section{Levodopa Formulations to benefit through the day}

Levodopa+Carbidopa Intestinal Gel (LCIG; Duodopa): aqueous supplied in $100 \mathrm{ml}$ cassettes containing $2000 \mathrm{mg}$ of levodopa, enough for a full day's treatment. The cassette attaches to a portable infusion pump that pumps the gel through a transabdominal tube connected to a percutaneousendoscopic gastrostomy (PEG) tube with the tip positioned in the proximal jejunum or duodenum. The patient remains ambulatory. LCIG is effective to reduce motor fluctuations and dyskinesia in advanced PD. ${ }^{18}$ Though expensive, invasive \& inconvenient, LCIG appears to be a reasonable alternative to DBS in appropriate patients.

As levodopa can only be absorbed across a short segment of the small intestine distal to the stomach, other L-DOPA formulations that is absorbed throughout the lower GI tract are being looked into, one of them is the XP21279.

\section{APOMORPHINE can be given in two ways:}

1. subcutaneous apomorphine injections "on demand;" or

2. continuous subcutaneous injection by means of a pump.

Apomorphine is a potent DA. Increases the duration of "on" phases and is effectively applicable to patients with motor fluctuations.

Neuroplasticity is the ability of brain's neurons to compensate for injury \& disease and adjust their activities in response to new situations \& changes in their environment. So brain retains the capacity to overcome some of the limitations caused by disease. Neuroplasticity is possible throughout the life span. Experience-dependent neuroplasticity is the basis of current day physical \& rehabilitative therapy.

\section{Parkinson's and exercise}

Progression of PD is not halted by exercise but the quality of life improves. Stronger more flexible muscles improves balance $\&$ helps to tackle motor symptoms with increased efficiency. Recent research shows that exercise seems to protect the dopamine-producing neurons helping them work better and survive for longer.

\section{How does exercise change the brain?}

Animal models of PD have shown exercise induced neuroplasticity. ${ }^{19}$

- $\quad$ Fisher et al. at the University of Southern California studied the brains of mice after exercise protocols parallel to human treadmill. Observations were:

1. Exercise group

a. utilized dopamine more efficiently in brain cells by modification of substantia nigra \& basal ganglia.

b. possessed less dopamine transporter, so dopamine signals lasted longer.

c. Had an up regulation of D2 receptor.

2. Exercise did not have any effect on amount of dopamine nor the number of neurons.

They also studied the D2 receptor in a subset of the human subjects who were within one year of diagnosis and not on any medications, using PET imaging \& found increased the number of $\mathrm{D} 2$ receptors.

- At the University of Pittsburgh researchers showed, exercise induces and increases neurotrophic factors, like GDNF (glial-derived)in animal models, which may be neuroprotective.

- $\quad$ Forced exercise[FE] is defined as active aerobic exercise where exercise rate is augmented mechanically to help the participant achieve and maintain a rate that is greater than their preferred voluntary rate of exercise. It is postulated that FE increases afferent signals from golgi tendon organs \& muscle spindles hence triggering the release of neurotrophic factors \& possibly dopamine.

- Exercise interventions in PD incorporate goal-based motor skill training to engage cognitive circuitry important in motor learning. Through instruction, feedback \& encouragement individuals become more cognitively engaged in learning movements that were automatic \& unconscious in their earlier life like swallowing, speaking, walking etc.. Goal based aerobic exercise has the potential to improve both cognitive \& automatic components of motor control in mild to moderate PD through experience-dependent neuroplasticity. ${ }^{20}$

- Some of the different types of exercise, include tai chi, tango dancing, Feldenkrais method, gym training and hydrotherapy, Lee Silverman Voice Treatment.

Deep Brain Stimulation[DBS] has become the surgical procedure of choice for PD for the following reasons:

- It does not involve destruction of brain tissue

- It is reversible 
- It can be adjusted as the disease progresses or adverse events occur

- Bilateral procedures can be performed without a significant increase in adverse events.

A better understanding of basal ganglia physiology and circuitry and improvements in surgical techniques, neuroimaging, and electrophysiology have allowed surgical procedures to be performed more accurately with lower morbidity.

Surgery for movement disorders previously involved predominantly destructive lesioning of abnormally hyperactive deep brain nuclei; however, the observation that high-frequency electrostimulation in the ventral lateral nucleus (VL) of the thalamus eliminates tremors in patients undergoing thalamotomy led to investigation of long-term DBS as a reversible alternative. Focus of movement disorder surgery are 3 key gray-matter structures: the thalamus, the globuspallidus, and the subthalamic nucleus (STN). Currently, the STN is the most commonly targeted site for PD.

\section{Targets for pharmacotherapy $\&$ beyond}

With loss of nigrostriatal neurones there is

1. dampening of direct pathway \&

2. release of indirect pathway

Net effect is an overactive GPi, strongly inhibiting the thalamus and causing a reduction in cortical activity, causing hypokinesia [Fig.-2].

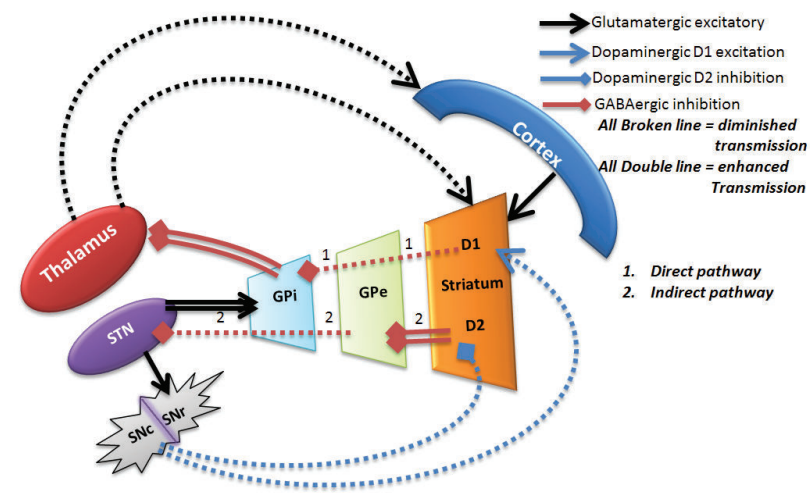

Fig-2 : Simplified basal ganglıa circuitry parkinson's disease - Hilaly et al.

The motor effects of early PD manifest when $>\mathbf{5 0 \%}$ dopaminergic neurons are damaged $\& \mathbf{8 0} \%$ dopamine is reduced. So before the symptoms appear the deficit is compensated by brain plasticity. Treatment at this early stage, is rewarding for the physician \& patient alike.

\section{Physicians resorts to}

I. dopamine precursor L-DOPA/carbidopa or

II. stimulate dopamine receptor subtypes with DAs[ropinirole, pramipexole] or

III. reduce the breakdown of dopamine with MAO-B inhibitors[selegiline and rasagiline].

Prof Kailash Bhatia, at the Institute of Neurology, London, are among those who are researching as to the timing of treatment initiation. Some trials suggest that people who start drug treatment soon after being diagnosed respond better than those who delay treatment and this may be related to brain plasticity. Dopamine replacement causes a quick improvement in motor function the short duration response[SDR]. XiaoxiZhuang et al. suggests dopaminedependent corticostriatal plasticity, and retention of such plasticity in the absence of dopamine, are the mechanisms underlying the long duration response[LDR] \& therapies that enhance the LDR could be more effective than those targeting the SDR, a form of disease modification ${ }^{21}$.

As PD ages there is progressive depletion of endogenous dopaminergic neurotransmission spreading throughout the CNS \& an increase in the exogenous supply of L-DOPA. Soon, an imbalance between dopamine receptor subtypes may occur \& the development of LID. Further adaptive changes of other neurotransmitters[glutamate, GABA, serotonin...] in the basal ganglia occur. The glutamatergic projections from the subthalamic nucleus $[\mathrm{STN}]$ to the GPi become overactive [Fig 2], which can damage any remaining dopaminergic neurons resulting in progression of PD symptoms including LID. Therefore research focus is shifting towards new nondopaminergic targets:

a. NMDA receptors mediate glutamatergic excitation in the striatum and STN. Amantadine is a weak noncompetitive NMDA receptor antagonist \& has shown success in PD and LID.

b. Metabotropic Glutamate Receptors(mGlu): trials with mGlu5 receptor antagonists, mavoglurant and dipraglurant, are ongoing in treating PD and LID. ${ }^{11}$

c. Ionotropic Glutamate Receptors are potential therapeutic targets for symptomatic management in PD and LID. ${ }^{11}$

d. Safinamide may provide benefit in PD through both MAO-B inhibition and inhibition of glutamate release. ${ }^{18}$

e. Adenosine2a(A2a) antagonists. An intriguing observation from animal studies is that coadministration of an A2a antagonists from the time dopaminergic therapy is begun might prevent the development of dyskinesia. ${ }^{18}$ 
f. Gene therapies not yet promising.

g. Transplantation of stem cells to replace degenerating dopaminergic neurons has so far had limited success in patients

Younger age at onset with resting tremor as a presenting symptom often predicts a benign course with a longer benefit to L DOPA. DA are commonly reserved for younger individuals ( $<65-70$ years) who are cognitively intact. When introducing DA, it is started at a low dose and escalated slowly. Patients on DA should be routinely asked about sleepiness, sudden onset of sleep, and ICD such as pathologic gambling, shopping, internet use, and sexual activity. Patients should not drive in case of undue sleepiness. When DA (with or without MAO-B inhibitor) no longer provides good control of motor symptoms, L-DOPA can be added. In contrast older age at onset, presenting with hypokinesia/rigidity, gait instability \& dementia predicts a less benign course \& poorer response to L-DOPA. For patients with cognitive impairment and those older than 70 years - who may be prone to adverse effects, such as hallucinations, from DA - and for those likely to require treatment for only a few years, L-DOPA is the primary symptomatic therapy.

NICE, Canadian \& other guidelines advocate that anticholinergics [e.g, trihexyphenidyl, benztropine] may be used as Second-line drugs for tremor only due to the propensity to cause neuropsychiatric side effects. If PD is accompanied by postural tremor $\beta$-blockade may be useful.

After long-term L-DOPA treatment fluctuation in response starts.

End of dose deterioration is signaled when the effect of $\mathrm{L}$ DOPA wanes off about 4 hours after the last dose. First approach is then to fine tune L DOPA therapy to provide more sustained dopamine levels:

1. Adding

- DA,

- catechol- $O$-methyltransferase (COMT) inhibitor,

- MAO-B inhibitor

Then attempting to lower L-DOPA dose.

2. Frequent L-DOPA dosing: smaller doses are administered more frequently. The time to wearing-off determines the interdose interval. In extreme cases using liquid levodopa, the dose can be titrated finely and administered every hour.

3. Increasing L-DOPA dose

\section{Switching from immediate-releaseto}

- $\quad$ sustained or extended release(CR) levodopa/carbidopa or
- levodopa/carbidopa/entacapone[COMT inhibitor] inhibit the peripheral metabolism of L-DOPA to 3-O methyldopa, prolonging L-DOPA half-life making it available for crossing the blood-brain barrier over a longer period. If dyskinesia starts, the L-DOPA dose is reduced. Entacapone is preferred to tolcapone [hepatotoxic].

Unless limited by peak-dose symptoms like dyskinesia or hallucinations, dopaminergic therapy should be increased until off time is eliminated. But soon the inevitable dyskinesia, freezing, camptocormia in combination with non-motor symptoms make life for the patient \& caregiver and prescribing for the physician a living hell. The ELLDOPA trial compared the use of three doses of an immediate release carbidopa/levodopa combination (in a 1:4 ratio) and placebo over 40 weeks in 361 drug naïve patients with early PD. There was an improvement in motor symptoms in all the levodopa treatment groups. Adverse effects (dyskinesia, hypertonia...) were increasingly associated with high-dose treatment and longer duration of treatment ${ }^{16}$. Many patients develop peak-dose dyskinesia. At this point, increasing dopamine stimulation may worsen dyskinesia, and decreasing dopamine stimulation may worsen PD motor signs and increase off time. The therapeutic window lies above the "on threshold" required to improve symptoms and below the threshold for peak-dose dyskinesia. The therapeutic window of L-DOPA soon narrows with a progressive decrease in the threshold for peak-dose dyskinesia such that minor increments in dose starts dyskinesia.

Use of Amantadine in PD with motor fluctuations \& dyskinesia is evidence based. Amantadine improves dyskinesia.

As PD progresses, adequate control of motor symptoms depends increasingly on continuous drug delivery. So extended release \& sustained release formulations may benefit the off time patients. LCIG is yet another option in practice over 30 or so countries, especially in advanced PD or LID but costs $>\$ 60,000 /$ year.

Botulinum toxin has emerged as an important therapeutic option alone or in combination to alleviate non-motor symptoms like sialorrhoea, pain etc. \& also motor symptoms like freezing, camptocormia though not yet advocated by evidence based guidelines. Gait freezing that occurs when PD is undertreated ('off' freezing, plus significant residual tremor, rigidity, or bradykinesia) may respond to an increase in dopaminergic replacement therapy. Gait freezing that occurs when the PD is well controlled ('on' freezing) is difficult to treat and may be worsened by increasing dopamine replacement therapy. 
Intermittent subcutaneous apomorphine may be considered for the reduction in 'off' time in patients with advanced Parkinson's disease.

When fine tuning the medical options fail to control motor fluctuations DBS is currently the surgical treatment of choice in advanced PD.

Non-motor symptoms are often missed \& not reported for various obvious reasons. So a routine screening technique should be practiced at follow-up visits. The authors find the

Non-motor symptoms questionnaire given in Annex 3 of the Scottish Intercollegiate Guidelines Network; Jan 2010.www.sign.ac.uk, useful.

Patients must be educated with information that is appropriate for their disease state and expected or ongoing challenges. It is important to provide information at the time of diagnosis about the condition, therapy and progression. Patient must feel that they are not alone. Must appreciate the importance of medication timing \& exercise. One person with PD who missed his medication stated: "I just kind of froze up...you have to find out the hard way [that that's] what the medication is doing to you" "16. The best time to educate the patient \& caregiver is in small amounts at the time of diagnosis \& subsequent follow-ups so they become adopted with hope. We must stress on managing the human with PD humanely. Giving too little information - "I was shocked in maybe 12 minutes of his total time seeing me, he diagnosed me with an illness and gave me no hope and told me to take some medicine. And then he dismissed me"16 can be shattering. And giving too much information may be confusing \& frightening, in my early years of clinical practice I remember telling my patient at the time of diagnosis that a tiny part of her brain is drying up, told her the consequences \& was about to start the prognosis but I looked into her face \& asked "are you scared" she replied "Now I am!'. She developed LID 10 years after the diagnosis $\&$ lived for a further two years. If nothing then we should never fail to give our patients hope.

Physical \& rehabilitative therapy cannot be overemphasized especially after ongoing concepts of experience based neuroplasticity. With focused motor skill training patients learn to better control the movements that were once automatic but now impaired like speaking, walking, balancing, swallowing etc.. Aerobics also improve the muscular blood supply, flexibility \& strength.

\section{Conclusion}

Dr. James Parkinson's essay, "The Shaking Palsy" written in 1817, narrates a disease later named by the French Neurologist Charcot as Parkinson's disease [PD]. The story of olfactory dysfunction, pain, depression, constipation, sleep disturbance followed a few years later by unilateral resting tremor, rigidity, bradykinesia eventually becoming bilateral with gait disorder, "mask-like" facial appearance, sialorrhoea \& the inevitable dyskinesia, freezing, dysautonomia, cognitive \& psychiatric embarrassment all have a telling decrease in the quality of life of the patient $\&$ the caregiver. The solution lies not in treating the disease but in managing the human suffering from PD aiming to fine tune pharmacotherapy to minimise adverse effects \& optimise quality of life. With the current knowledge \& evidence, the state of the art management is an individualized, multidisciplinary approach entertaining neuroplasticity. Customized care addresses the non-motor problems which at times are more disabling than the motor problems. Age of onset should serve as a soft guide in choosing initial drugs early in the disease. Though L DOPA remains the anchor drug $\&$ albeit very promising in early disease yet the late fluctuations \& LID limit its usefulness. The narrow therapeutic window of L-DOPA late in the disease makes it a depressing scenario. Even with the modern advances an effective management is unavailable, so there must be further research and trends in Research aim are:

1. Ways to

a. sustained antiparkinsonian benefit through the day,

b. ameliorate or prevent dyskinesia, and

c. slow or prevent the progression of the disease

2. To solve the basal ganglia circuitry mystery to better target nondopaminergic neurotransmission

3. Better physical, speech \& rehabilitative strategies

4. The relative efficacy of various anti-parkinsonian drug groups should be studied in different age cohorts

5. Dopamine dependent plasticity

6. Role of the cerebellum in Parkinson's disease

Conflict of Interests: The authors report no conflicts of interest in this work

\section{References:}

1. A. Berardelli et al.EFNS/MDS-ES recommendations for the diagnosis of Parkinson's disease.European Journal of Neurology 2013;20:16-34

2. C E Clarke, Clinical Review Parkinson's disease. BMJ. 2007 September 1;335(7617):441-445.

3. Canadian Guidelines on Parkinson's Disease.Canadian Journal of Neurological Sciences.2012 July; Volume 39 Number 4 (Supplement 4)

4. Meara J, Bhowmick B, Hobson P. Accuracy of diagnosis in patients with presumed Parkinson's disease. Age Ageing 1999;28:99-102. 
5. National Institute for Health and Clinical Excellence. Parkinson's disease: diagnosis and management in primary and secondary care London: NICE, 2006; clinical guideline $35: 11$.

6. David J Pedrosa, Review: management of Parkinson's disease. Neuropsychiatric Disease and Treatment 2013:9 321-340.

7. John G. Nutt, M.D., and G. Frederick Wooten, M.D. N Engl J Med 2005; 353: 1021-1027.

8. John G. Nutt et al.The On-Off Phenomenon in Parkinson's Disease - Relation to Levodopa Absorption and TransportNEngl J Med 1984; 310: 483-488.

9. E. Lugingeretal.Beneficial effects of amantadine on L-dopainduced dyskinesias in Parkinson's disease .Movement Disorders.E. LugingerVol 15, Issue 5, Sept 2000; 873-878.

10. Richard Mayeux, M.D.et al.Reappraisal of Temporary Levodopa Withdrawal (Drug Holiday) in Parkinson's Disease.NEngl J Med 1985; 313:724-728

11. FabrizioGasparinietal.Review Article: Metabotropic Glutamate Receptors for Parkinson's Disease Therapy.Parkinson's Disease 2013; Article ID 196028. http:/ /dx.doi.org/10.1155/2013/196028.

12. J. E. Ahlskog and M. D. Muenter, "Frequency of levodoparelated dyskinesias and motor fluctuations as estimated from the cumulative literature," Movement Disorders, vol. 16, no. 3, pp. 448-458, 2001.

13. Ariane Park and Mark Stacy, Review ArticleDopamineInduced Nonmotor Symptoms of Parkinson's Disease.
Parkinson's Disease 2011, Article ID 485063. Hindawi Publishing Corporation.

14. Anne Marie Bonnet et al.Nonmotor Symptoms in Parkinson's Disease in 2012: Relevant Clinical Aspects,Parkinsons Dis. 2012; 2012: 198316.

15. Arbouw ME et al.Glycopyrrolate for sialorrhea in Parkinson disease: a randomized, double-blind, crossover trial.Neurology. 2010 Apr 13;74(15):1203-7

16. Diagnosis and pharmacological management of Parkinson's disease; A national clinical guideline; Scottish Intercollegiate Guidelines Network; Jan 2010. www.sign.ac.uk

17. Bradley Voytek. Emergent Basal Ganglia Pathology within Computational Models. JNEUROSCI.2255-06.2006; 26(28): 7317-7318; doi: 10.1523.

18. Robert A. Hauser.Future Treatments for Parkinson's Disease: Surfing thePD Pipeline.International Journal of Neuroscience, 121, 53-62, 2011.

19. Hirsc MA et al. Exercise \& neuroplasticity in persons living with Parkinson's disease. Eur J Phys Rehab Med. 2009 Jun;45(2):215-29.

20. Giselle M Petzinger et al. Exercise enhanced neuroplasticity targeting motor \& cognitive circuitry in Parkinson's disease. The Lancet Neurology. Jul 2013; vol 12(7):716-726.

21. Zhuang, X. et al. (2013)The role of neuroplasticity in dopaminergic therapy for Parkinson disease. Nat. Rev. Neurol. doi:10.1038/nrneurol.2013.57.

22. Tao $\mathrm{Wu}$ et al. The Cerebellum in Parkinson's Disease. Brain. 2013;136(3):696-709. 